\title{
Automatic Feedback on Cognitive Load and Emotional State of Traffic Controllers
}

\author{
Mark A. Neerincx ${ }^{1,2}$, Maaike Harbers ${ }^{1}$, Dustin $\operatorname{Lim}^{1}$, and Veerle van der Tas ${ }^{1}$ \\ ${ }^{1}$ Delft University of Technology, Mekelweg 4, 2628 CD, Delft, The Netherlands \\ ${ }^{2}$ TNO, P.O. Box 23, 3769 ZG Soesterberg, The Netherlands \\ mark.neerincx@tno.nl, M.Harbers@tudelft.nl
}

\begin{abstract}
Workload research in command, information and process-control centers, resulted in a modular and formal Cognitive Load and Emotional State (CLES) model with transparent and easy-to-modify classification and assessment techniques. The model distinguishes three representation and analysis layers with an increasing level of abstraction, focusing on respectively the sensing, modeling, and reasoning. Fuzzy logic and its (membership) rules are generated to map a set of values to a cognitive and emotional state (modeling), and to detect surprises of anomalies (reasoning). The models and algorithms allow humans to remain in the loop of workload assessments and distributions, an important resilience requirement of human-automation teams. By detecting unexpected changes (surprises and anomalies) and the corresponding cognition-emotion-performance dependencies, the CLES monitor is expected to improve team's responsiveness to new situations.
\end{abstract}

Keywords: resilience engineering, workload, affective computing, electronic partners, traffic management.

\section{Introduction}

Train traffic management has to deal with a complex internal and external environment, in which all kind of disturbances can appear with possibly cascading effects. Automation changed, and will further change, the operational control \& supervision processes [1]. Automation has become an actor in these processes. Human and automation have to deal jointly with possible conflicts and dependencies between solutions, means and resources during the disturbance responses. Two types of research questions can be distinguished, centering (1) on the actual process of dealing with anomalies or (2) on the realization of a resilient human-automation team. For the first type, an example research question for train traffic control, is "how to improve distributed situation awareness and balance workload across operators through sharing of information on (i) what remote others are doing, (ii) how they are progressing with their tasks, and (iii) how their task progression affects the common goal". For the second type, an example research question for train control is "how to improve the competencies and skills of operators through situated feedback on (i) their physical and mental condition, (ii) their relationships with team-members, and (iii) their work attitude and motivation". 
A knowledge-based approach is required to answer these questions and to develop corresponding methods for achieving resilience, e.g., by providing support on performance, behavior, intention, task progression, and mental and physical condition of (remote) actors to increase coordination among actors and to subsequently allocate resources flexibly [2]. Such support improves the capabilities to mutually empower the human and automation for disturbance anticipation, monitoring, responding, and learning. For such mutual empowerment, we aim at ePartners that help humans in complex dynamic environments, and as such improve resilience [3, 4]. Such ePartners should have knowledge of the (momentary) capacities of the traffic controller and the current task and context in which she or he operates, and be able to assess the fit of these capacities to the task demands. To enhance resilience, the ePartners and human operators should be able to share, complement and correct each other's assessment of the workload distribution among humans and automatons.

There is a rich history and enormous amount of research on workload in the human factors domain (e.g., [5]) and on emotion in the affective computing domain (e.g., [6]). However, workload models, techniques and applications are diverse with different levels of granularity. Moreover, there is a lack of models that both (i) are understandable and accessible for the operators themselves, and (ii) formalize the interrelationships between the cognitive and affective processes for realistic complex settings. In other words, for real-world traffic control settings, there is not yet a sound, self-explaining formal model to automatically assess and guide cognitive and affective processes coherently. This paper presents the incremental development of such a model, to be implemented in a support tool for real-time balancing of cognitive and affective load.

\section{Background}

For several years, we conducted research to derive a transparent, coherent and concise workload model from established human factors theories and empirical studies in realistic process control settings. Taking an incremental development approach, we aimed at a modular model that can be fed or instantiated with information from both "machine and human sensors" [7]. The modules should allow for human-machine sharing of knowledge, for job design, mission planning and dynamic (real-time) workload allocation. This research was conducted in the train traffic control, naval ship center control, and space domain.

First, it was concluded that classical workload analyses for train traffic control mainly assessed the Time Occupied (TO) of train dispatchers, disregarding the cognitive demands or the work. To address these demands, a Cognitive Task Load (CTL) analysis was developed that assesses the required Level of Information Processing (LIP) (among others, based on the Knowledge, Rule and Skillframework of Rasmussen, [8]). Simple and routine tasks evoke more efficient (i.e., a lower level of) information processing, whereas complex and new tasks require more extensive and intensive processing. The new CTL-analysis method proved to provide better assessments than the "old" method: the identification of inadequate workload 
distribution in control posts, the reveal of context-dependencies and the proposal of a standard [9].

Subsequently, a third CTL-factor was added to the model, Task-Set Switches (TSS), to address the work demands of process control operations that may have to respond to unforeseen events or alarms immediately [10]. These demands entail both the responses on a single event after a long period of indifference and the responses on a large number of, almost co-occurring, alarms. The 3-dimensional model identifies specific types of CTL-states (properties): overload, under-load, vigilance, cognitive lock-up and optimal load [11]. The corresponding CTL-analysis method provided adequate predictions of the task load and identified negative effects on operator performance of under- and overload situations in a naval ship control center [12]. Furthermore, applying a "Naïve Bayesian network" for predicting performance from the CTL-values, provided performance estimations with $86 \%$ and $74 \%$ accuracy for a, respectively, high-fidelity simulator and real ship control center [13].

However, this CTL-model does not address the affective processes of work, which have a major impact on the performance in our high-demand application domains. Therefore, an Emotional State (ES) model was being constructed that complements the CTL-model. The combined model is called the Cognitive Load and Emotional State (CLES) model.

\section{The CLES Model}

In this section we explain the internal model of the CLES tool. After providing a general overview, we give more detailed discussions on the modeling of cognitive load and emotional state, respectively. We will pay attention in particular to the use of fuzzy logic for emotional state modeling, enabling to reason (i) in a transparent way (ii) with variables that have a truth value between 0 and 1 [14].

\subsection{Three Layers of CL and ES Analysis}

The monitor consists of three layers in which cognitive load and emotional state are represented and analyzed. The layers have an increasing level of abstraction. The activities performed in each layers are the following.

1. Sensing of cognitive load and emotional state

2. Modeling of cognitive load and emotional state

3. Reasoning about cognitive load and emotional state

In the first layer, information about the operator being monitored is perceived. More specifically, the CLES tool perceives observables regarding the operator's physical state and the tasks he performs. In the second layer, these observables are used to model cognitive load and emotional state. This results in a cognitive load value and an emotion classification, respectively. In the third layer, the CLES tool reasons about the cognitive load and emotional state of the operator, e.g. to detect surprising changes or rare combinations. 


\subsection{Cognitive Load Modeling}

Cognitive load modeling in the CLES tool is based on Neerincx et al.'s model of cognitive task load (CTL) that was introduced in Section 2 of this paper. In layer 1 of the CLES tool, the tasks that the operator performs are observed in order to calculate an operator's cognitive load based on the CLT model. The following information is required for each task that the operator performs: the type of task, starting time, and finishing time.

As discussed in Section 2, the CTL model consists of three components: time occupied (TO), level of information processing (LIP), and task set switching (TSS). In layer 2 of the CLES tool, a value is calculated for each of these components for a given time frame, based on the observables represented in layer 1. TO is calculated by taking the percentage of time the operator was performing tasks in that time frame. Layer 2 contains domain knowledge representing the level of information processing for all tasks in that domain. LIP is calculated by taking the average level of information processing of all tasks performed in the time frame. TSS is the total number times the operator switched tasks in the time frame. Subsequently, these three values are combined to determine the cognitive load of the operator in that time frame (see [15] for the set of formula).

The cognitive load of an operator is continuously determined in layer 2 . This value, changing over time, forms the input for layer 3 of the CLES tool. In this layer, sudden changes in cognitive load are detected (the next version will also detect risks for vigilance and cognitive lock-up). Furthermore, the combination of an operator's cognitive load and emotional state is being monitored and reasoned about.

\subsection{Emotional State Modeling and Fuzzy Logic}

Emotional state modeling is based on the Pleasure-Arousal-Dominance (PAD) model [16]. This model quantifies emotional state according to three dimensions: pleasure, arousal and dominance. An option is to leave out the dimension of dominance, resulting in the Valence-Arousal (VA) model which still produces a useful classification of emotional states

In layer 1 of the CLES tool, information is collected to determine an operator's emotional state. There are different ways to assess someone's valence and arousal. Valence can, for example, be assessed by recording and classifying someone's facial expressions, or by using sensors that measure facial muscle activity. Arousal is usually assessed by measuring someone's hart rate or hart rate variability, and galvanic skin response.

In layer 2, fuzzy logic is used to determine an operator's emotional state based on the physiological measures observed in layer 1 (see also [17]). We chose to use fuzzy logic because it enables reasoning with multiple statements that are partially true, rather than statements that are merely truth or false. Partial truth of a statement is represented by a value that ranges between 0 and 1 . In fuzzy logic, a collection of such values forms a fuzzy set, and in a fuzzy inference process, rules are applied to this set to produce a new fuzzy set. For example, fuzzy sets of heart rate and galvanic 
skin response can be combined to create a new fuzzy set of arousal, and fuzzy sets of arousal and valence can be combined to a new fuzzy set of emotional state. An example of a fuzzy inference rule is: 'if galvanic skin response is high and heart rate is medium high then arousal is high'. Finally, when all fuzzy sets are combined, the final fuzzy set undergoes a process of defuzzification to produce a single, most likely outcome. In the CLES tool this final outcome represents the operator's emotional state.

Like cognitive load, an operator's emotional state is continuously determined in layer 2, and used for the reasoning process in layer 3 of the CLES tool. The outcome of this reasoning can be, for instance, a warning for the operator or his superior when the operator is heavily burdened.

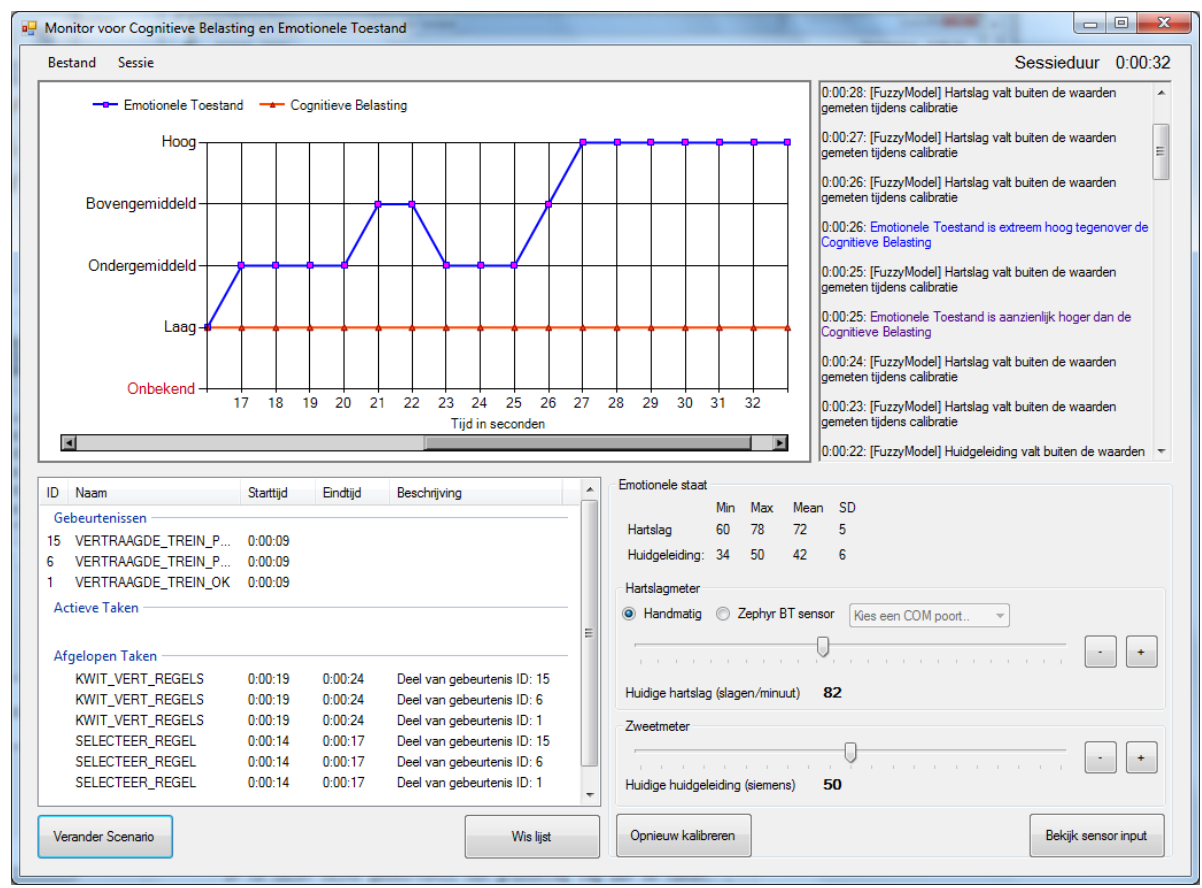

Fig. 1. Screenshot of the CLES monitor while running

\section{The CLES Tool}

Figure 1 shows a screenshot of the CLES tool while running. The display is divided into four components. The right and left parts in the lower half of the display give an overview of the operator's task performance and physical measures, respectively. These parts correspond to layer 1 in the CLES tool. The upper left part of the display shows the respective cognitive load (red line) and emotional state (blue line) of the operator. When the CLES tool is running, the graph is moving. This part corresponds to the output of the cognitive load and emotional state modeling in layer 2 of the 
CLES tool. The upper right part of the displays shows messages that are the result of the reasoning component in layer 3 of the CLES tool. One of the messages is, for example, that emotional state is extremely high with regard to cognitive load.

In its current condition, the CLES tool perceives information about the heart rate and galvanic skin response of the operator. Input of heart rate information can be manually, through a slider, or automatically through a Zephyr BT sensor. Input of galvanic skin response is manually. The nature and number of input values can easily be adapted. Based on the physical measures, through fuzzy logic, the operator's level of arousal is determined. In the current CLES tool, thus, arousal is used to indicate emotional state.

Table 1. Example scenario of train breakdown

\begin{tabular}{|l|l|}
\hline Scenario & Explanation \\
\hline task(communication, 10, 100, DT) & $\begin{array}{l}\text { Conversation with driver of } \\
\text { defective train }\end{array}$ \\
\hline task(communication, 110, 200, DT) & $\begin{array}{l}\text { Conversation with traffic control } \\
\text { officer }\end{array}$ \\
\hline $\begin{array}{l}\text { task (select rule, 200, 203, DT) } \\
\text { task (find train, 205, 220, DT) } \\
\text { task(automatic program off, 220, 223, DT) } \\
\text { task(deselect rule, 223, 225, DT) }\end{array}$ & $\begin{array}{l}\text { Cancel automatic control of } \\
\text { defective train }\end{array}$ \\
\hline task(recall signal, 230, 250, DT) & Recall signal at switch \\
\hline $\begin{array}{l}\text { task(select rule, 260, 263, DT) } \\
\text { task(find train, 265, 285, DT) } \\
\text { task(automatic program off, 285, 288, DT) } \\
\text { task(manually process rule, 290, 350, DT) } \\
\text { task(automatic program on, 350, 353, DT) } \\
\text { task(deselect rule, 355, 358, DT) }\end{array}$ & Cancel automatic control \\
\hline task(communicate, 380, 440, DT) & Conversation with mechanic \\
\hline $\begin{array}{l}\text { task(select rule, 440, 443, DT) } \\
\text { task(find train, 445, 465, DT) } \\
\text { task(manually process rule, 470, 530, DT) } \\
\text { task(automatic program on, 530, 533, DT) } \\
\text { task(deselect rule, 535, 538, DT) }\end{array}$ & Recover automatic control \\
\hline
\end{tabular}

Task performance information is generated by a simulator in the present state of the CLES monitor. The simulator simulates events such as a train that is delayed or a switch that does not work, and based on that, a list of tasks that the traffic control operator needs to perform. Each task is annotated with a beginning and an end time. An example scenario of a train that breaks down is provided below. The CLES tool has knowledge about the level of knowledge processing associated to these tasks, and uses this knowledge and the input from the simulator to determine the operator's cognitive load. 
Table 1 presents an example scenario about a train that breaks down. Information is represented in the following way: $\operatorname{task}\left(\mathrm{id}, \mathrm{t}_{\text {start }}, \mathrm{t}_{\text {end }}\right.$, event $)$, where id refers to task $\mathrm{id}, \mathrm{t}_{\text {start }}$ to the starting time of the task, $\mathrm{t}_{\text {end }}$ to the end time of the task, and event to the event for which the task was performed. In the scenario below, all tasks were performed in the context of event DT, a defective train. The left column shows the tasks of the operator, and the right column provides an explanation of the tasks.

\section{$5 \quad$ Conclusions and Discussion}

Based on prolonged research in different domains, we have identified, combined and formalized models of cognitive and affective load with transparent and easy-tomodify classification and assessment techniques. Fuzzy logic and its (membership) rules are generated to map a set of values to an emotional state, and to detect surprises of anomalies. The models and algorithms allow humans to remain in the loop of workload assessments and distributions, an important resilience requirement of human-automation teams. The current tool provides basic feedback, which is expected to improve human-automation team's awareness about the adequacy of the workload distributions and possibilities for improvement. By detecting unexpected changes (surprises and anomalies) and the corresponding cognition-emotionperformance dependencies, the CLES monitor can improve team's responsiveness to new situations, i.e., its resilience.

Current research focuses on the development of ePartners that are continuously informed by the CLES monitor. Based on this knowledge and other available information (e.g. from a user model), the ePartner will improve the feedback and provide advice at the individual and team level. The advice concerns, for example, the provision of insight in person's own functioning to improve his self-efficacy, and proposals for task (re)allocation to improve team performance [18].

Acknowledgement. This research was conducted within the RAILROAD project and is supported by ProRail and the Netherlands organization for scientific research (NWO) (under grant 438-12-306).

\section{References}

1. Ferreira, P.N., Balfe, N.: The contribution of automation to resilience in rail traffic control. In: Harris, D. (ed.) EPCE 2014. LNCS (LNAI), vol. 8532, pp. 469-480. Springer, Heidelberg (2014)

2. Hollnagel, E., Woods, D.D., Leveson, N. (eds.): Resilience engineering: concepts and percepts. Ashgate Publishing Limited, Hampshire (2006)

3. Neerincx, M.A., Grant, T.: Evolution of Electronic Partners: Human-Automation Operations and ePartners During Planetary Missions. Journal of Cosmology 12, 3825-3833 (2010)

4. Neerincx, M.A.: Situated Cognitive Engineering for Crew Support in Space. Personal and Ubiquitous Computing 15(5), 445-456 (2011)

5. Wickens, C.D., Hollands, J.G., Parasuraman, R., Banbury, S.: Engineering Psychology \& Human Performance, 4th edn. Prentice-Hall (2012) 
6. Picard, R.: Affective computing. MIT Press, Cambridge (1997)

7. Neerincx, M.A., Lindenberg, J.: Situated cognitive engineering for complex task environments. In: Schraagen, J.M.C., Militello, L., Ormerod, T., Lipshitz, R. (eds.) Naturalistic Decision Making and Macrocognition, pp. 373-390. Ashgate Publishing Limited, Aldershot (2008)

8. Rasmussen, J.: Information Processing and Human-Machine Interaction: An Approach to Cognitive Engineering. Elsevier, Amsterdam (1996)

9. Neerincx, M.A., Griffioen, E.: Cognitive task analysis: harmonizing tasks to human capacities. Ergonomics 39(4), 543-561 (1996)

10. Kiesel, A., Steinhauser, M., Wendt, M., Falkenstein, M., Jost, K., Philipp, A.M., Koch, I.: Control and interference in task switching-A review. Psychological Bulletin 136(5), 849-874 (2010)

11. Neerincx, M.A.: Cognitive task load design: model, methods and examples. In: Hollnagel, E. (ed.) Handbook of Cognitive Task Design, ch. 13, pp. 283-305. Lawrence Erlbaum Associates, Mahwah (2003)

12. Grootjen, M., Neerincx, M.A., Veltman, J.A.: Cognitive task load in naval ship control centres: from identification to prediction. Ergonomics 49, 1238-1264 (2006)

13. Neerincx, M.A., Kennedie, S., Grootjen, F., Grootjen, M.: Modelling Cognitive Task Load and Emotion for Adaptive Maritime Interfaces. In: Schmorrow, D.D., Estabrooke, I.V., Grootjen, M. (eds.) Foundations of Augmented Cognition: Neuroergonomics and Operational Neuroscience. Proceedings of the 5th International Conference of the Augmented Cognition. LNCS (LNAI), pp. 260-269. Springer, Heidelberg (2009)

14. Zadeh, L.A.: Fuzzy sets, fuzzy logic, and fuzzy systems: selected papers, vol. 6. World Scientific (1996)

15. Harbers, M., Aydogan, R., Jonker, C.M., Neerincx, M.A.: Sharing Information in Teams: Giving Up Privacy or Compromising on Team Performance? In: Proceedings AAMAS 2014, Paris, France, May 5-9 (2014)

16. Mehrabian, A.: Pleasure-arousal-dominance: A general framework for describing and measuring individual differences in temperament. Current Psychology 14(4), 261-292 (1996)

17. Mandryk, R.L., Atkins, M.S.: A fuzzy physiological approach for continuously modeling emotion during interaction with play technologies. International Journal of HumanComputer Studies 65(4), 329-347 (2007)

18. De Greef, T.E., Arciszewski, H.F.R., Neerincx, M.A.: Adaptive Automation based on an Object-Oriented Task Model: Implementation and Evaluation in a Realistic C2 Environment. Journal of Cognitive Engineering and Decision Making 4, 152-173 (2010) 